\title{
Mediation analysis to unravel mechanisms underlying association between platelet transfusion and postoperative delirium
}

\author{
Bingwen Zhang ${ }^{1}$ and Zhongheng Zhang $^{2^{*}}$ (D) \\ See related Research by Rudiger et al. http://ccforum.biomedcentral.com/articles/10.1186/s13054-016-1445-8
}

We read with great interest the study by Rudiger and colleagues [1]. It is a retrospective study investigating risk factors of postoperative delirium in patients undergoing cardiopulmonary bypass surgery. The authors collected many perioperative potential risk factors and then performed univariate and multivariate analyses to investigate their association with the development of delirium. This is the standard method for risk factor analysis [2]; however, it has long been criticized for a substantial proportion of false positive results. Let us suppose that an investigator chooses 20 factors but none of them has an association with the outcome; regression analyses may obtain positive results a substantial number of times. A method to address this problem is to validate the regression model in an independent cohort (e.g., external validation). However, with the limited sample size and single center nature of the study, this validation cohort was not used.

An alternative approach to such data-driven analysis is hypothesis-driven analysis. Data-driven analysis is performed without the prior hypothesis that it examines the association between risk factors and outcome. Positive associations are reported depending on the results of statistical results. On the other hand, the hypothesis-driven approach is to make a hypothesis before data collection, and data are then used to test whether the hypothesis is supported by the empirical data. An interesting method is the mediation analysis [3]. In the study cited, we could hypothesize that duration of cardiopulmonary bypass is an initiator which causes delirium via the inflammatory pathway, and that inflammatory responses can be measured by platelet indices and $\mathrm{C}$ reactive protein [4]. Other pathways also include the tissue hypoxia that can be characterized by lactate and hemodynamics. Once a specific pathway is determined, empirical data can be utilized to test whether the theory is supported by data. Mediation analysis is suitable for the analysis. Alternatively, if the sample size is large enough, the structural equation model can also be helpful to unravel complex relationships between risk factors and outcomes.

Our previous study found that lactate was associated with postoperative occurrence of acute kidney injury [5]. The possible underlying mechanism is tissue hypoxia during and after operation. It is possible that tissue hypoxia may have universal adverse ef-

* Correspondence: zh_zhang1984@hotmail.com

2Department of Emergency Medicine, Sir Run-Run Shaw Hospital, Zhejiang University School of Medicine, No. 3, East Qinchun Road, Hangzhou 310016, Zhejiang Province, China

Full list of author information is available at the end of the article 
fects on organ functions. Delirium is a type of acute organ dysfunction involving the central nervous system, and thus can be affected by hypoxia. Consistent with this hypothesis, the study showed that lactate levels on intensive care unit (ICU) day 1 were significantly associated with delirium $(p=0.008)$.

\section{Authors' response}

\section{A. Rudiger, M. Schubert, B. Seifert, D. R. Spahn and D. Bettex}

We have recently published in Critical Care that delirium occurred in $26 \%$ of consecutive ICU patients undergoing cardiac surgery [1]. In this study, patients with and without delirium differed significantly in disease severity scores, intraoperative variables, postoperative laboratory parameters, and the utilization of ICU resources. Focusing on intraoperative variables, cardiopulmonary bypass duration, low mean arterial blood pressure, low hemoglobin levels, low body temperature, high noradrenaline requirements, and transfusion of red blood cells and platelets were significant intraoperative risk factors. In the multivariate analysis, only platelet transfusions remained independent. This suggests that the interplay of different events during cardiac surgery might promote postoperative delirium.

Regarding our findings, Zhang and Zhang highlight in their letter that repetitive statistical testing bears the risk of false positive results [6]. In addition, the authors mention that a hypothesis-driven study has advantages over a data-driven analysis. Mediation analysis is another suggested option.

We thank the authors for these comments and would like to add that a retrospective analysis can only reveal strong associations but does not give answers on causality. Nevertheless, findings from observational studies remain interesting and clinically important for the following reasons: First, observational studies represent the real world whereas randomized controlled trials often exclude important patient groups. As a consequence, well-designed observational studies do not systematically overestimate the magnitude of the associations between exposure and outcome as compared with the results of randomized controlled trials of the same topic [7]. Second, the realization of randomized controlled trials in critically ill patients is challenging, partly due to ethical and administrative constraints, and partly because of the complex interplay between underlying diseases, pathophysiological deteriorations, and therapeutic interventions [8].

Abbreviation

ICU: Intensive care unit

Authors' contributions

BZ and ZZ conceived the idea. BZ and ZZ drafted the manuscript. All authors read and approved the final manuscript.

Competing interests

The authors declare that they have no competing interests.

\section{Author details}

${ }^{1}$ Department of Critical Care Medicine, Jinhua Municipal Central Hospital, Jinhua Hospital of Zhejiang University, 351\#, Mingyue street, Jinhua 321000, Zhejiang Province, China. ${ }^{2}$ Department of Emergency Medicine, Sir Run-Run Shaw Hospital, Zhejiang University School of Medicine, No. 3, East Qinchun Road, Hangzhou 310016, Zhejiang Province, China.

Published online: 28 October 2016

References

1. Rudiger A, Begdeda H, Babic D, Krüger B, Seifert B, Schubert M, et al. Intraoperative events during cardiac surgery are risk factors for the development of delirium in the ICU. Crit Care. 2016;20:264.

2. Zhang Z. Multivariable fractional polynomial method for regression model. Ann Transl Med. 2016;4:174-4.

3. Van der Weele TJ. Mediation analysis: a practitioner's guide. Annu Rev Public Health Annual Reviews. 2016:37:17-32

4. Zhang Z, Pan L, Deng $\mathrm{H}, \mathrm{Ni} \mathrm{H}, \mathrm{Xu}$ X. Prediction of delirium in critically ill patients with elevated C-reactive protein. J Crit Care. 2014;29:88-92.

5. Zhang Z, Ni H. Normalized lactate load is associated with development of acute kidney injury in patients who underwent cardiopulmonary bypass surgery. PLoS One. 2015;10:e0120466.

6. Zhang B, Zhang Z. Mediation analysis to unravel mechanisms underlying association between platelet transfusion and postoperative delirium. Crit Care 2016, in press. doi:10.1186/s13054-016-1513-0.

7. Concato J, Shah N, Horwitz RI. Randomized, controlled trials, observational studies, and the hierarchy of research designs. N Engl J Med. 2000;342: 1887-92.

8. Vincent $J \mathrm{~L}$, Singer M. Critical care: advances and future perspectives. Lancet. 2010;376:1354-61. 